ISSN 1823-626X

Journal of Fundamental Sciences

available online at http://jfs.ibnusina.utm.my

\title{
Sylvester-type matrices for sparse resultants
}

\author{
Shamsatun Nahar Ahmad ${ }^{1}$ and Nor'aini Aris ${ }^{2 *}$ \\ ${ }^{1}$ Faculty of Computer and Mathematical Sciences, Universiti Teknologi MARA(Johor), km 12 Jalan Muar, 85009 Segamat, Johor, Malaysia. \\ ${ }^{2}$ Department of Mathematics, Faculty of Science, Universiti Teknologi Malaysia, 81310 Skudai, Johor Bahru Johor, Malaysia. \\ Received 18 March 2010, Revised 4 May 2010, Accepted 17 May 2010, Available online 21 June 2010
}

\begin{abstract}
The resultant matrix of a polynomial system depends on the geometry of its input Newton polytopes. Therefore for sparse inputs, the matrix is lower in dimension. The aim of the study is to infer conditions on the class of polynomial systems that can give a resultant matrix whose size is minimized, that is an optimal or Sylvester-type sparse resultant matrix. From the work of Emiris, the 'incremental algorithm' has been claimed to produce optimal matrices for the class of multi-homogeneous (or multigraded) systems of special structure. Cyclic polynomial systems for n-root problems also fall under this classification. We have applied the Maple multires package to obtain Sylvester-type matrices for some examples. The ultimate aim of the study is to verify whether the multigraded systems constitute to the only class of polynomial systems that can give sparse resultant optimal matrix; hence giving a necessary and sufficient condition for producing exact sparse resultants.
\end{abstract}

| multihomogeneous polynomial systems | optimal Sylvester-type matrices | sparse resultant matrix |

( 2010 Ibnu Sina Institute. All rights reserved. http://dx.doi.org/10.11113/mjfas.v6n1.173

\section{INTRODUCTION}

The problem of solving a system of nonlinear polynomial equations $f_{1}=f_{2}=\ldots=f_{m}=0$ over a field $X$ such that the system has a solution, arise in many application domains. For instance, in algebra, mechanics, kinematics, robotics, structural molecular biology, logic, geometric and solid modelling, image understanding and vision have been well-documented [1, 6, 12, 19, 21]. It is for this reason that techniques of resultant for solving such applications as above have received considerable research attention due to its efficientness and robustness to certain problems (e.g. [2, 4, 7, 9, 16, 22]). Many of these studies have reported on the success of the method being used in the applications.

For each $i=1,2, \ldots, m$, let $f_{i}=\sum_{a_{j} \in A_{i}} c_{i, a_{j}} \mathbf{x}^{a_{j}}$ that $A_{i}=\left\{a_{1}, a_{2}, \ldots, a_{s}\right\} \subseteq Z^{n}$ is the set of exponent vectors or support $a_{i}$ for $f_{i}$. Resultant is a single polynomial (i.e. an irreducible polynomial) derived from the original system of polynomial equations that encapsulates the solution to the system (refer to e.g. $[13,14,17,20]$ ). A matrix is sparse if it consists of entries which are mostly zeroes. A sparse resultant matrix is a sparse matrix such that the nonzero coefficients consist of terms in the polynomial system.

Corresponding author at: Faculty of Computer and Mathematical Science UiTM Johor, Km 12 Jalan Muar, 85009 Segamat Johor, Malaysia

E-mail addresses: shams551@johor.uitm.edu.my (Shamsatun Nahar Ahmad)
The size of the resultant matrix is determined by the input polytopes, named Newton polytopes, denoted as $Q_{i}=\operatorname{conv}\left(A_{i}\right) \subseteq X^{n}$ where $A_{i}$ denotes the support of the ith polynomial of the system [6].

Sparse resultant matrices are of any of different types: Sylvester-type matrices (e.g. [6,7,13]), where the polynomial coefficients fill in the nonzero entries of the matrix; Bezout-type matrices, where the coefficients of the Bezoutian associated to the input polynomials fill in the nonzero entries of the matrix (e.g. [6, 18]) and hybrid matrices (e.g. [5, 10] ). This study focuses on Sylvester-type matrices for sparse resultant.

In Emiris [12], two effective methods for constructing sparse resultant matrices were described based on discrete geometric operations, namely the subdivisionbased method and the incremental algorithm. The first approach (called greedy version), relies on a mixed subdivision of the Minkowski sum; $Q=Q_{0}+\ldots+Q_{n}$, which generalizes the sparse elimination theory [2, 3, 4]. The theory uses combinatorial and discrete geometry to exploit the structure of a given polynomial system. The methods had been applied in computing mixed volume and formulating sparse resultant matrices for problems in three different areas of research, namely camera motion from point matches, cyclic molecules and Stewart platform kinematics.

The main idea is to decrease the size of the matrix which defines the resultant. For certain class of systems a single matrix whose determinant equals the resultant can be 
constructed; that is, a Sylvester type formula can be derived. In addition, the resultant matrix is optimal when its dimension is minimal, that is when the size of the matrix is equal to the degree of the resultant so that any entry is either zero or is an input coefficient. The incremental approach is able to derive an optimal Sylvester-type matrix for certain problems [13].

Almost all polynomial systems modelled in applications are sparse. Therefore, it is necessary to encounter sparse polynomials, which involve only exponents lying in a finite set [8]. In addition, it is necessary to utilize the sparsity structure of polynomials which are prevalent in almost all real life problems, yielding a generalized resultant, named the sparse resultant.

Several studies have established necessary and sufficient conditions that can give exact resultants using the Dixon resultant formulation [5, 6, 18, 19, 22]. Based on the ideas that have been presented in the analysis of the Dixon case, the research is conducted with the aim of inferring and characterizing sparseness conditions on the structure of the Newton polytopes and support interiors of the class of polynomial systems that produce exact resultants for sparse systems. In this preliminary work, Maple multires package is used to compute the resultant matrices of some multivariate polynomial systems. The class of each polynomial system is first determined. Comparisons of the size of the resultant matrix with the mixed volume of the Newton polytopes of the corresponding multigraded system are made to check whether the matrix obtained is optimal. An optimal matrix formula is expected from the class of multigraded systems.

\section{EXPERIMENTAL}

As a preliminary work, Maple multires package is used to compute the resultant matrices of nonlinear polynomial systems. The greedy variant of the subdivision based algorithm is named after Pederson [3]. The algorithm starts with a single row, corresponding to some integer point, and proceeds iteratively by adding new rows and columns. For a given set of rows, the column set comprises all columns required to express the row polynomials. For a given set of columns, the rows are updated to correspond to the same set. The algorithm continues by adding rows and corresponding columns until a square matrix has been obtained. The algorithm computes resultant matrices of the greedy version.

In this paper, the greedy algorithm is applied to several multihomogeneous systems of three polynomials with two variables. Multihomogeneous structure is a first step away from the classical theory of homogeneous systems towards fully exploiting arbitrary sparse structure [11] and are encountered in several areas including geometric modelling [5, 19, and 21].

A polynomial $f \in X(\mathbf{x}, c)$ is called multihomogeneous of type $\left(l_{1}, l_{2}, \ldots, l_{r} ; k_{1}, k_{2}, \ldots, k_{r}\right)$ if $f=\sum c_{\alpha} \mathbf{x}_{1}^{k_{1}} \mathbf{x}_{2}^{k_{2}} \ldots \mathbf{x}_{r}^{k_{r}}$, where $\mathbf{x}_{i}^{k_{i}}=\left(x_{i, 1}^{p_{1}} \ldots x_{i, l_{i}+1}^{p_{l_{i}+1}}\right), x_{i, l_{i}+1}^{p_{l_{1}+1}}$ is the homogenizing variable and $p_{1}+p_{2}+\ldots+p_{l_{i}}+p_{l_{i}+1}=k_{i}$. A multihomogeneous polynomial of type $\left(l_{1}, \ldots, l_{r} ; d_{1}, \ldots, d_{r}\right)$ is called multigraded if for each $i=1,2, \ldots, r$, either $l_{i}=1$ or $d_{i}=1[15]$, and it is an unmixed system of $n+1$ generic multigraded polynomials in $n$ variables, where $\sum_{i=1}^{r} l_{i}=n$ [6].

In this paper we illustrate the results of the size of the resultant matrices of the greedy version for certain types of multigraded systems. The matrix size is compared to the mixed volume of the corresponding Newton polytopes of the system, applying Theorem 1 and Theorem 2 given below.

Theorem 1 (Bernstein's Theorem)[8].

If $f_{1}, f_{2}, f_{3}$ are three generic polynomials in two variables, then the number of solutions of $f_{1}(x, y)=f_{2}(x, y)=f_{3}(x, y)=0$ in $\left(C^{*}\right)^{2}$, equals the mixed volume, $M V\left(Q_{1}, Q_{2}, Q_{3}\right)$ where $Q_{i}=\operatorname{conv}\left(A_{i}\right)$.

Theorem 2 [2]. The sparse resultant is separately homogeneous in the coefficients $c_{i}$ of each $f_{i}$ and its degree in these coefficients equals the mixed volume of the other $n$ Newton polytopes, $\quad M V_{-i}=M Y\left(Q_{1}, \ldots, Q_{-1}, Q_{1+1}, \ldots, Q_{n+1}\right)$, $i=1, \ldots, n+1$ that is $\operatorname{deg}_{i} R=M$ (i).

Sturmfels and Zelevinsky showed that every multigraded system has at least one Sylvester type formula for its sparse resultant . Emiris and Canny proved that, for any multigraded system, an initial vector can be defined from the sequence $l_{k}$, the Newton polytopes, and a scaled down copy of these polytopes where the scaling has occurred by a different factor for every group of $l_{k}$ coordinates. Consequently, the first matrix constructed by the resultant matrix algorithm has determinant equals to the sparse resultant of the system. More precisely, for every multigraded system, an optimal matrix formula can be constructed [13, pg 25].

\section{RESULTS \& DISCUSSION}

The computation of mixed volume is obtained using formula [24],

$$
M V(Q)=\sum_{I \subset\{1,2, \ldots, n\}}(-1)^{n-\# I} \operatorname{Vol}\left(\sum_{i \in I} Q_{i}\right) .
$$

Considering over constrained system with two variables, equation (1) reduces to $M V\left(Q_{1}, Q_{2}\right)=\operatorname{Vol}\left(Q_{1}+Q_{2}\right)-\operatorname{Vol}\left(Q_{1}\right)-\operatorname{Vol}\left(Q_{2}\right)$. Here "Vol" is referred to usual area measure of plane Euclidean geometry.

Example 1. Consider the following generic polynomial system with generic coefficients,

$$
\begin{aligned}
& f_{1}=a_{11}+a_{21} y+a_{31} y^{2}+a_{41} x+a_{51} x y+a_{61} x^{2} \\
& f_{2}=a_{12}+a_{22} y+a_{32} y^{2}+a_{42} x+a_{52} x y+a_{62} x^{2} \\
& f_{3}=a_{13}+a_{23} y+a_{33} y^{2}+a_{43} x+a_{53} x y+a_{63} x^{2}
\end{aligned}
$$




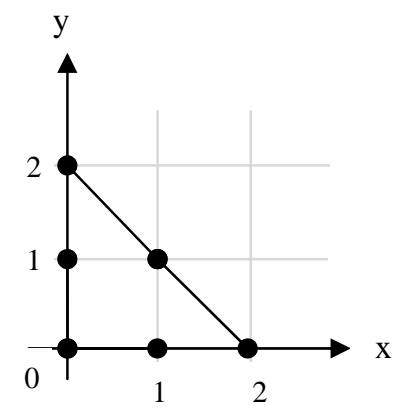

$\mathrm{A}_{i}=\{(0,0),(1,0),(1,1),(0,1),(2,0),(0,2)\}, \mathrm{i}=1,2,3$

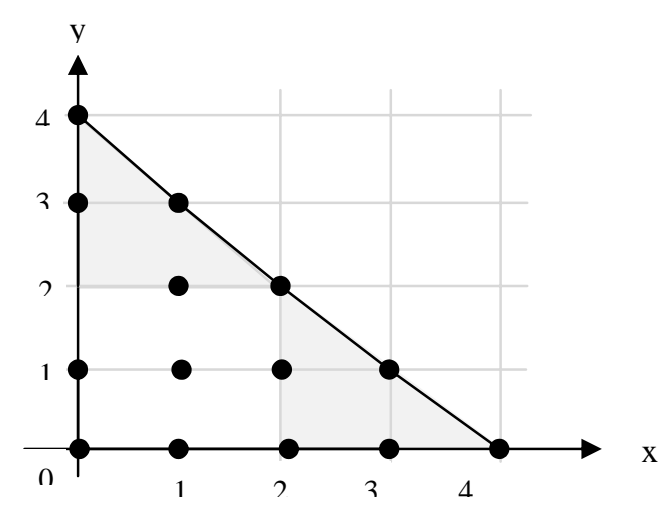

$\mathrm{Q}_{1}+\mathrm{Q}_{2}=\operatorname{conv}\left(\mathrm{A}_{1}+\mathrm{A}_{2}\right)$

Figure 1: Newton polytopes (convex hull) of each polynomial for multihomogeneous polynomial of type $(1,1 ; 2,2)$ and Minkowski sum of any two-fold and subdivision

The system is a multigraded polynomial of type $(1,1 ; 2,2)$. The Newton polytopes or the convex hull of each polynomial of this system and its Minkowski sum of subdivision can be depicted respectively, by Figure 1 .

The system is unmixed and the mixed volume of any two of the polynomials is 4 . Hence the resultant matrix has at least 4 rows coming from each polynomial, implying that it must have 12 rows to be the exact resultant matrix or optimal matrix of Sylvester-type. In particular, by mixed volume computation of its Newton polytopes, the degree of the sparse resultant is 12 . However, the Maple multires package, through function spresultant, gives a sparse ma- trix of size 14. An optimal matrix of size 12 can actually be derived since the system is multigraded.

Example 2. Consider the polynomials

$f_{1}=a_{11} x^{2}+a_{21} x+a_{31} x y+a_{41}, f_{2}=a_{32} x y+a_{42}, f_{3}=a_{23} x+a_{33} x y+a_{43}$

The system is multigraded of type $(1,1 ; 2,1)$. The convex hull of the supports of this system can be viewed in Figure 2. Consequently, its Minkowski sum of two-fold and subdivision is shown in Figure 3.

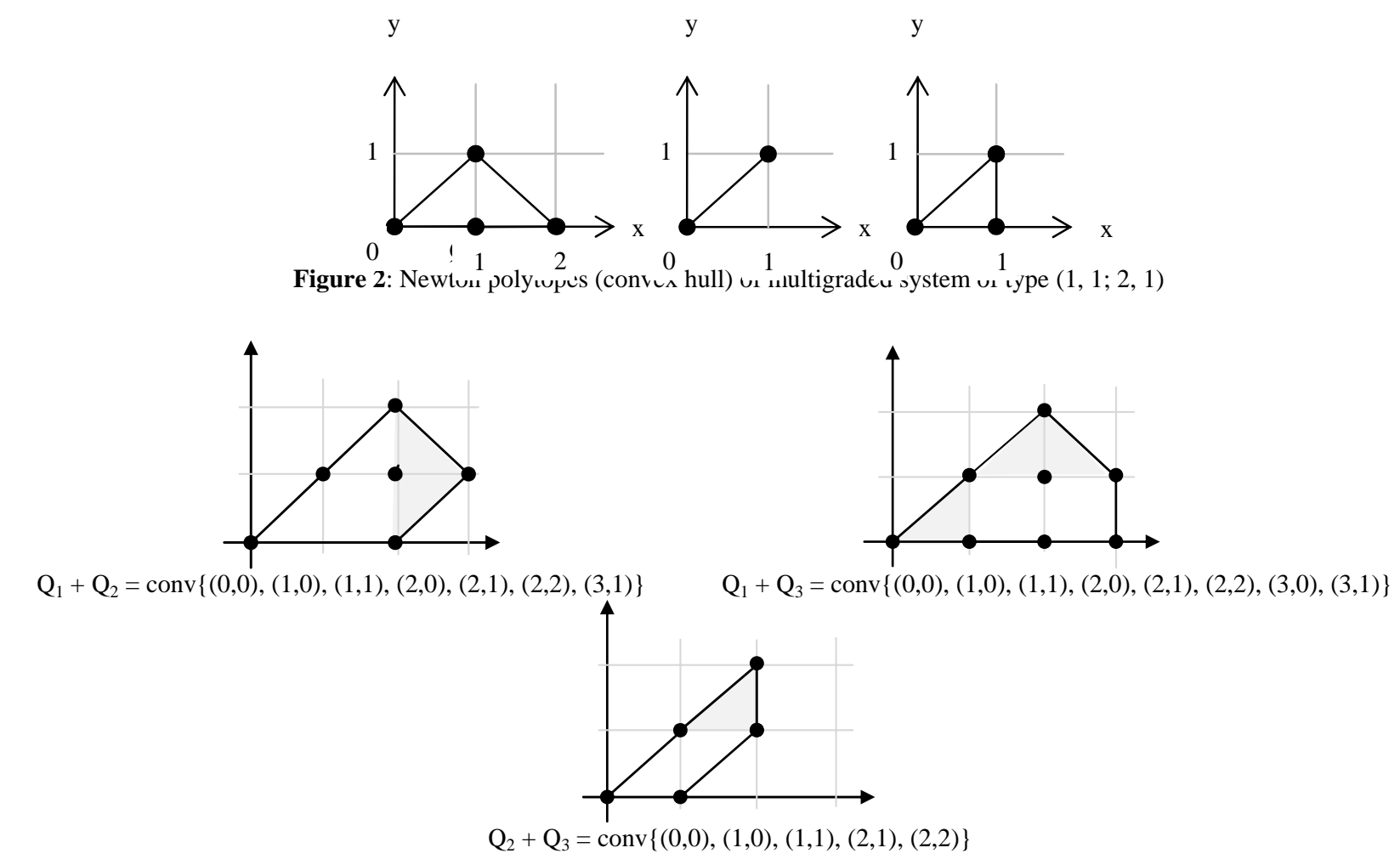

Figure 3: Minkowski sum of two-fold of multigraded system of type $(1,1 ; 2,1)$ and subdivision 
Its sparse resultant matrix given by the Maple multires package is a $5 \times 5$ matrix. The size of this matrix equals mixed volumes of two-fold polynomials given by $M V\left(Q_{1}, Q_{2}\right)+M V\left(Q_{1}, Q_{3}\right)+M V\left(Q_{2}, Q_{3}\right)=2+2+1=5$. Since the degree of the resultant is 5 , the construction gives an optimal or Sylvester-type matrix. In addition, if a system with this mixed supports is solvable, Theorem 1 implies that it has five nonzero roots. The results of the computation have also shown that the mixed volume expresses the sparseness of the system by taking the sublattice of $\mathrm{Z}^{n}$ generated by the Minkowski sum of all supports.

Example 3. Consider the polynomials

$$
\begin{aligned}
& f_{i}=c_{1 i} x^{2} y+c_{2 i} x^{2} z+c_{3 i} x^{2} t+c_{4 i} x s y+c_{5 i} x s z+c_{6 i} x s t+ \\
& c_{7 i} y s^{2}+c_{8 i} z s^{2}+c_{9 i} s^{2} t \text { for } i=1,2,3
\end{aligned}
$$

which is multigraded of type $(1,2 ; 2,1)$. The convex hull of the supports of this system can be viewed as in Figure 4. The system is unmixed with variable subset $x$, s and $y, z, t$. The homogenizing variables are $s$ and $t$ in the respective block. The convex hull for the unmixed supports of the system is shown in Figure 4.

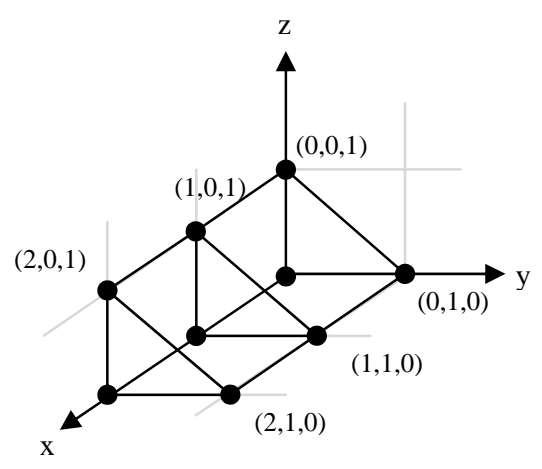

Figure 4: Newton polytopes (convex hull) of each polynomial for multigraded polynomial of type $(1,2 ; 2,1)$

To apply multires package the technique of u-resultant is applied to form an over constraint system given by

$$
\begin{aligned}
& f_{i}=c_{1 i} x^{2} y+c_{2 i} x^{2} z+c_{3 i} x^{2} t+c_{4 i} x s y+c_{5 i} x s z+c_{6 i} x s t+ \\
& c_{7 i} y s^{2}+c_{8 i} z s^{2}+c_{9 i} s^{2} t, \text { for } i=1,2,3 \\
& f_{4}=u_{0}+u_{1} x+u_{2} y+u_{3} z .
\end{aligned}
$$

Then multires gives a sparse matrix of size 59 . Whereas, the sparse resultant's total degree is the sum of mixed volumes, $M V\left(Q_{1}, Q_{2}\right)+M V\left(Q_{1}, Q_{3}\right)+M V\left(Q_{2}, Q_{3}\right)+M V\left(Q_{1}+Q_{4}\right)+$ $M V\left(Q_{2}+Q_{4}\right)+M V\left(Q_{3}+Q_{4}\right)=6+6+6+3+3+3=27$.

We illustrate the Newton polytopes of this system in Figure 5. The shaded region is a translation of $Q_{1}, Q_{2}, Q_{3}$, and $Q_{4}$ respectively. These shaded regions are not counted in the computation of mixed volume. Since the system is ultigraded, an optimal matrix of size 27 can actually be derived.
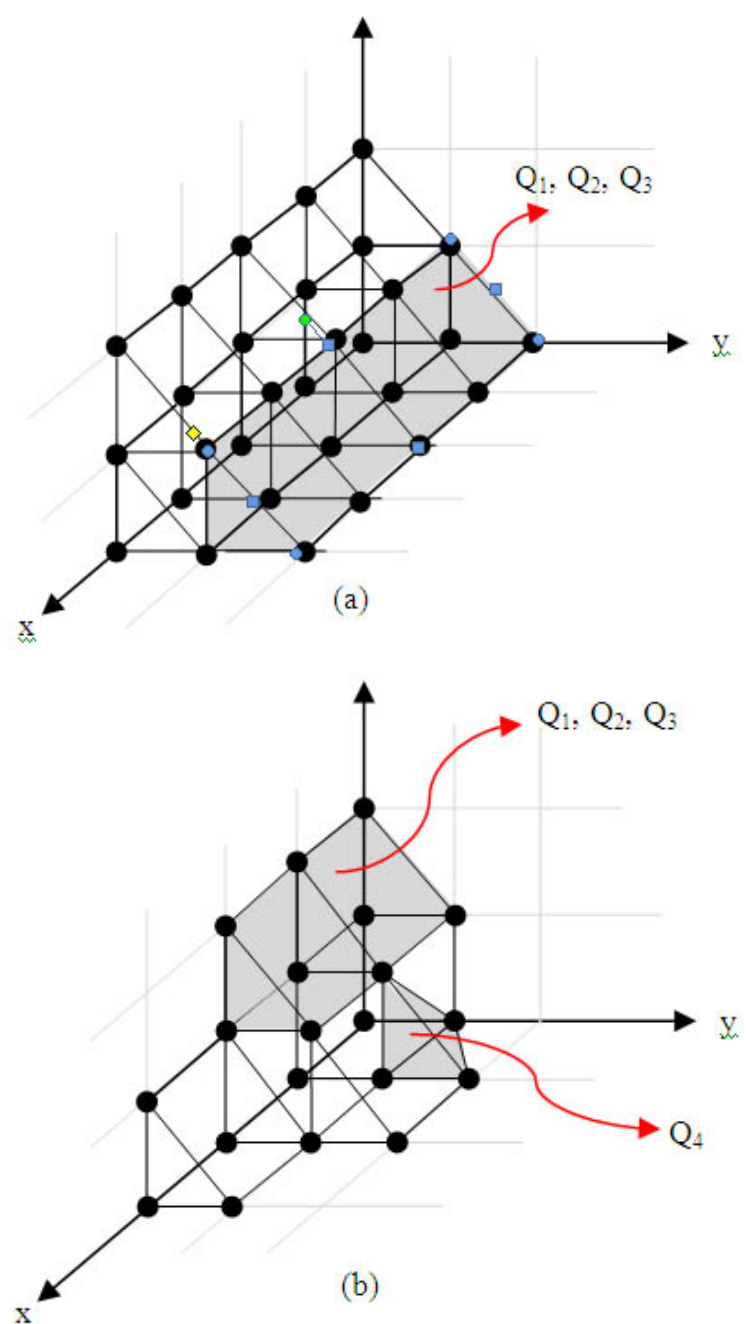

a) Minkowski sum of $\left(\mathrm{Q}_{1}+\mathrm{Q}_{2}\right),\left(\mathrm{Q}_{1}+\mathrm{Q}_{3}\right)$ and $\left(\mathrm{Q}_{2}\right.$ $+\mathrm{Q}_{3}$ ) and its subdivision

b) Minkowski sum of $\left(\mathrm{Q}_{1}+\mathrm{Q}_{4}\right),\left(\mathrm{Q}_{2}+\mathrm{Q}_{4}\right)$ and $\left(\mathrm{Q}_{3}\right.$ $\left.+\mathrm{Q}_{4}\right)$ and its subdivision

Figure 5: Minkowski sum of two-fold of multigraded system of type $(1,2 ; 2,1)$ and subdivision

\section{CONCLUSION AND FURTHER WORK}

We have illustrated the implementation of the greedy version algorithm on some examples of multigraded systems of types $(1,1 ; 2,2),(1,1 ; 2,1)$ and $(1,2 ; 2,1)$ respectively. The first type is an unmixed system. After homogenizing variables, the system can be written as $f_{i}=a_{i 1} t^{2} z^{2}+a_{i 2} y t z^{2}+a_{i 3} y^{2} z^{2}+a_{i 4} x t^{2} z+a_{i 5} x y t z+a_{i 6} x^{2} t^{2}$ with $\mathbf{x}_{1}^{2}=y^{p_{1}} t^{p_{2}}$ and $\mathbf{x}_{2}^{2}=x^{p_{1}} z^{p_{2}}$. The greedy implementation does not give an optimal matrix even though we expect an optimal matrix can be constructed from the class of multigraded systems. Investigation on other types of multigraded systems in two variables with one homogenizing variable from each class, and with various 
total degrees in each monomial, needs to be carried out. For systems with more than two variables, $l_{i} \neq 1$, the condition that the total degree of each monomial to be 1 , i.e. $d_{i}=1$ needs to be satisfied. In addition, the properties for unmixed systems need to be independently explored from the mixed cases.

The methods of constructing optimal matrices from multigraded systems need to be studied in the effort of finding a general algorithm that can construct a sparse resultant for every problem, extending to the case of other classes of multihomogeneous systems as well as systems for specific application problem. A resultant matrix for a system may not be optimal, but obtaining a matrix whose size is minimal is desirable in order to reduce the presence of extraneous factors in the formulation and construction of sparse resultant matrices, leading to the solutions of polynomial equations using the resultant approach.

\section{ACKNOWLEDGEMENT}

We acknowledge financial supports from Universiti Teknologi MARA (Johor), Malaysia and Universiti Teknologi Malaysia through the Fundamental Research Grant (FRGS) vot no. 78529.

\section{REFERENCES}

[1] J. F. Canny, The Complexity of Robot Motion Planning, doctoral dissertation, (1993), MIT, Cambridge, Mass.

[2] J. F. Canny, and I. Z. Emiris, In Proceeding of AAECC, In Lect. Notes in Comp. Science, Berlin, Springer-Verlag, (1993) 89 - 104.

[3] J. F. Canny, and P. Pederson, Tech. Report 1394, (1993), Computer Science Department, Cornell University.

[4] J. F. Canny, and I. Z. Emiris, Journal of the ACM, Vol. 47, 3, (2000) 417 - 451.

[5] E. W. Chionh, R. Goldman, and M. Zhang, In Proc. $8^{\text {th }}$ IMA Conf. Math. Of Surfaces, (1998) $193-212$.

[6] A. D. Chtcherba, A New Sylvester-type Resultant Method based on the Dixon-Bezout Formulation, Ph.D Thesis, Computer Science, University of New Mexico, New Mexico, (2003).

[7] A. D. Chtcherba, and D. Kapur, In Proceedings of ISSAC,2002, Lille, France.

[8] D. Cox, J. Little, and D. O’Shea, Using Algebraic Geometry. in Graduate Texts in Mathematics, Springer-Verlag, New York, 185 (1998).

[9] R. M. Corless, P. M. Gianni, and B. M. Trager, Proceedings of ISSAC, New York, ACM Press, (1997) $133-140$.

[10] C. D'Andrea, and I. Z. Emiris, Proceedings of ISSAC'01, Ontario, Canada. ACM Press, (2001) $24-31$.

[11] A. Dickenstein, and I. Z. Emiris, ISSAC, Lille, France, ACM Press,(2002) 46 - 54.

[12] I. Z. Emiris, Sparse Elimination and Applications in Kinematics, Doctoral Thesis, Computer Science Division, University of California., Berkeley, 1994.

[13] I. Z. Emiris, and J. F. Canny, J. Symbolic Computation, 20(2) (1995) 117-149.

[14] I. Z. Emiris, and V. Y. Pan, In proceed ACM ISSAC’97, Hawaii, July 21-21, ACM New York, (1997) 189 - 196.

[15] I. Z. Emiris, and B. Mourrain, Journal of Symbolic Computation, 28(1-2) (1999) 3-43.

[16] I. Z. Emiris, and A. Mantzaflaris, Technical Report 355881, INRIA, Sophia-Antipolis, France, (2009).

[17] I. M. Gelfand, M. M. Kapranov, and A. V. Zelevinski, Math.Theory Appl., Birkhauser, Boston, (1994).

[18] D. Kapur, and T. Saxena, ISSAC’95 Montreal Canada, ACM, (1995) 187 - 194.

[19] T. Saxena. Efficient variable elimination using resultants, Doctoral Thesis, Computer Science Department, State University of New York., Albany., College of art and sciences, (1997).

[20] B. Sturmfels, B. "Sparse Elimination Theory”, In Computation Algebraic Geometry and Commut. Algebra, D. Eisenbud and L. Robbiano, eds., Cortona, Italy. Proc, Symp. Math. Xxxiv, June (1991) $264-298$.

[21] M. Zhang, Topics in Resultants and Implicitization. PhD thesis, Dept. Comp. Science, Rice University, Houston, Texas, 2000.

[22] M. Zhang, and R.Goldman. Proc of the ISSAC, St Andrews, Scotland. ACM Press, (2000). 